\title{
Questionable Effectiveness of the QuantiFERON-TB Gold Test (Cellestis) as a Screening Tool in Healthcare Workers
}

\author{
Sumanth Gandra, MD, MPH; William S. Scott, MD, MPH; Vijaya Somaraju, MD, MPH; Huaping Wang, PhD; \\ Suzanne Wilton, APN, CNP; Michelle Feigenbaum, RN
}

\begin{abstract}
овjective. The Center for Disease Control and Prevention recommends the use of QuantiFERON TB Gold test (QFTG; Cellestis) in all situations where the tuberculin skin test (TST) is used. In November 2007, our institution replaced its screening-tool TST with the QFTG in-tube assay (QFT-GIT) for annual screening of latent tuberculosis infection among healthcare workers (HCWs). This study evaluated the effectiveness of QFT-GIT test as screening tool in HCWs at our institution.
\end{abstract}

METHODS. This retrospective study reviewed medical records of all HCWs who underwent screening with QFT-GIT from January 2008 through December 2008.

RESUlts. Among the 6,530 HCWs screened with QFT-GIT from January through December 2008, 287 had a positive test result. Of the 287, 123 had positive TST results in the past, meaning that 164 HCWs had newly diagnosed latent tuberculosis infection by QFT-GIT. Of the $164 \mathrm{HCWs}, 135$ were retested by QFT-GIT and were simultaneously tested with TST within 4 weeks after the initial QFT-GIT. Of these 135 HCWs, 66 reverted to negative results and results remained positive for 69 with QFT-GIT. Only 2 HCWs had a positive TST result.

CONCLUSIONS. The disagreement between QFT-GIT and TST results and the high reversion rate with QFT-GIT raise concerns about the effectiveness of QFT-GIT as a sole screening test in HCWs.

Infect Control Hosp Epidemiol 2010; 31(12):1279-1285

Screening healthcare workers (HCWs) for latent tuberculosis infection (LTBI) and active tuberculosis (TB) is well known infection control practice in the hospitals across the United States. Despite its drawbacks (ie, cross-reactivity with bacille Calmette-Guérin [BCG] and nontuberculous mycobacteria), the tuberculin skin test (TST) has been traditionally used for many years for detection of LTBI in HCWs. ${ }^{1}$ Recently, in vitro $\mathrm{T}$ cell-based assays called interferon (IFN) $-\gamma$ release assays (IGRAs) were developed to overcome the limitations of TST. These assays work on the premise that $\mathrm{T}$ cells previously sensitized to TB antigens produce high levels of IFN- $\gamma$ when re-exposed to the same mycobacterial antigens. ${ }^{2}$ Two such tests include the QuantiFERON-TB Gold assay (QFT-G; Cellestis) and T-SPOT.TB (Oxford Immunotec) enzyme-linked immunosorbent sport assay. With the QFT-G, blood samples are mixed with antigens and controls. The antigens include mixtures of synthetic peptides representing 2 Mycobacterium tuberculosis proteins, ESAT-6 and CFP-10, which are not found in BCG vaccine and many nontuberculous mycobacterial species. After incubation of the blood with antigens for 16-24 hours, the amount of IFN- $\gamma$ is measured. ${ }^{3}$ The major advantages of the QFT-G include its high specificity and that the results are unaffected by BCG vaccination. ${ }^{4}$ The other advantages are that it requires a single patient visit, it is not subject to reader bias, and it does not boost responses measured by subsequent tests, which can happen with TST.

In 2005, the Centers for Disease Control and Prevention (CDC) recommended the use of the QFT-G test in all situations in which a TST is used, including screening of HCWs. ${ }^{5}$ In November 2007, our institutional occupational health program replaced its TST screening tool with the QuantiFERON TB Gold in-tube assay (QFT-GIT; Cellestis) for annual screening of LTBI among HCWs. The QFT-GIT is a newer-generation QFT-G test that measures IFN- $\gamma$ production by T cells during in vitro stimulation with 3 peptides of the M. tuberculosisspecific antigens ESAT-6, CFP-10, and TB7.7. The performance of the QFT-G among HCWs has been studied in other countries ${ }^{6,7}$; however, there are limited data on its performance among HCWs in the United States. ${ }^{8}$ Therefore, we evaluated the effectiveness of the QFT-GIT test as screening tool at our institution.

\section{METHODS}

\section{Design}

This retrospective chart review study was approved by the University of Illinois College of Medicine at Peoria Institutional Review Board. We collected data from the medical

University of Illinois College of Medicine at Peoria (S.G., W.S.S., V.S., H.W.), and OSF St. Francis Medical Center (S.W., M.F.), Peoria, Illinois.

Received March 22, 2010; accepted June 14, 2010; electronically published October 27, 2010.

(C) 2010 by The Society for Healthcare Epidemiology of America. All rights reserved. 0899-823X/2010/3112-0011\$15.00. DOI: 10.1086/657336 


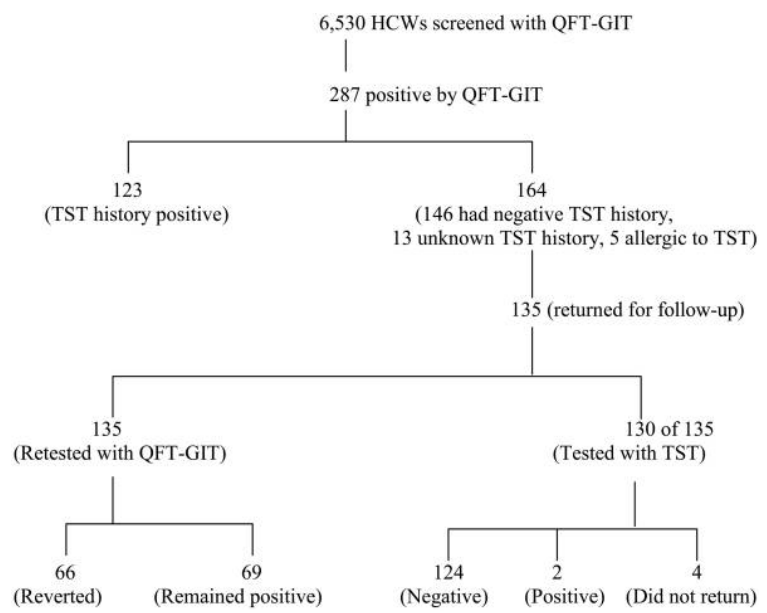

FIGURE 1. Flow diagram for 6,530 healthcare workers (HCWs) screened for latent tuberculosis infection with the QuantiFERON-TB Gold In-Tube (QFT-GIT; Cellestis) test. TST, tuberculin skin test.

records for all HCWs who underwent the QFT-GIT screening from January 1, 2008, to December 31, 2008. This 1-year data was reviewed and analyzed. The study was conducted at the occupational medicine section of OSF Saint Francis Medical Center, an academic tertiary care center and a major teaching affiliate of the University of Illinois College of Medicine at Peoria. The institution has more than 5,000 HCWs, most of them born in the United States. Fewer than $1 \%$ of HCWs were born overseas and are from areas where TB is highly endemic. All HCWs previously received a routine annual LTBI screen with a TST. However, in November 2007, on the basis of CDC guidelines, our institution replaced the TST with the QFT-GIT. The test was processed at our institution's system laboratory in accordance with the manufacturer's specifications. With QFT-GIT, whole blood is drawn into 3 precoated tubes ( 2 control tubes and $1 \mathrm{~TB}$ antigen tube). One of the controls has nil antigen, which serves as negative control; the other has a mitogen protein, which serves as positive control. The TB antigen tube contains 3 peptides specific to $M$. $t u$ berculosis: ESAT-6, CFP-10, and TB7.7. After incubation of the blood with antigens for 16-24 hours, the amount of IFN$\gamma$ is measured by enzyme-linked immunosorbent assay. The amount of IFN- $\gamma$ released is determined by subtracting the amount in the nil from the amount in the ESAT-6-, CFP10-, TB7.7-, or mitogen-stimulated plasma. The QFT-GIT test result is considered positive if the IFN- $\gamma$ response level is at least $0.35 \mathrm{IU} / \mathrm{mL}$ over the nil concentration. Nil concentrations of at least $8.0 \mathrm{IU} / \mathrm{mL}$ and mitogen differences of less than $0.5 \mathrm{IU} / \mathrm{mL}$ were considered indeterminate on the basis of the manufacturer's guidelines. The occupational medicine section initiated the routine QFT-GIT test on November 12 , 2007. Interestingly, within 6 weeks, an unexpected high number of HCWs had positive QFT-GIT test results: 11 HCWs had positive results. These HCWs had not had a positive TST result in the past. Because of the high positive conversion rate for LTBI with QFT-GIT, the Occupational Medicine section developed a protocol.

In accordance with the protocol, HCWs with an initial positive QFT-GIT test (QFT-GIT1) result underwent evaluation to exclude active TB infection by history, physical examination, and chest radiography. Their health records were checked for previous TST status. If the TST history ( $h$ TST) was negative or unknown or if the person was allergic, and if active TB infection had been excluded, the HCW underwent repeated testing with QFT-GIT (QFT-GIT2) and simultaneously with TST. The majority of the HCWs received these tests within 4 weeks of QFT-GIT1 at the same system laboratory. HCWs with positive QFT-GIT1 test results and positive $h$ TST results did not undergo repeated testing. Similarly, no additional tests were performed for HCWs with a negative QFT-GIT1 test result. The 1-step TST was done with Tubersol, in accordance to the CDC guidelines. A 2-step TST was done if the HCWs had not been previously tested with TST.

\section{Statistical Methods}

The conversion rate for the LTBI was calculated as a proportion of HCWs screened who had positive QFT-GIT1 results and had a negative, unknown, or allergic $h \mathrm{TST}$ result. Additional testing with QFT-GIT2 was performed for HCWs who had positive QFT-GIT1 and negative, unknown, or allergic $h$ TST results. The reversion rate was calculated as the proportion of HCWs who had a positive QFT-GIT1 result but a negative QFT-GIT2 result. The mean IFN- $\gamma$ response level for HCWs whose results remained positive (positive QFT-GIT1 and QFT-GIT2 results) was calculated and compared with those whose results reverted (positive QFT-GIT1

TABLE 1. Demographic Characteristics of 135 Healthcare Workers (HCWs) Who Underwent Repeated Testing

\begin{tabular}{lc}
\hline Feature & No. (\%) of HCWs \\
\hline Sex & $123(91.0)$ \\
Female & $12(9.0)$ \\
Male & \\
Country of birth & $127(94.0)$ \\
$\quad$ Area of nonendemicity ${ }^{\mathrm{a}}$ & $8(6.0)$ \\
Area of high endemicity & \\
TB exposure & $2(1.5)$ \\
BCG vaccination & \\
Positive & $3(2.3)$ \\
Unknown & $5(3.7)$ \\
Negative & $127(94.0)$ \\
TST history & \\
Negative & $130(96.3)$ \\
Unknown & $2(1.4)$ \\
Allergy & $3(2.3)$ \\
\hline
\end{tabular}

Note. BCG, bacille Calmette-Guérin; TB, tuberculosis; TST, tuberculin skin test.

a Areas of nonendemicity include the United States.

${ }^{\mathrm{b}}$ High-endemicity areas include India, Pakistan, and Mexico. 


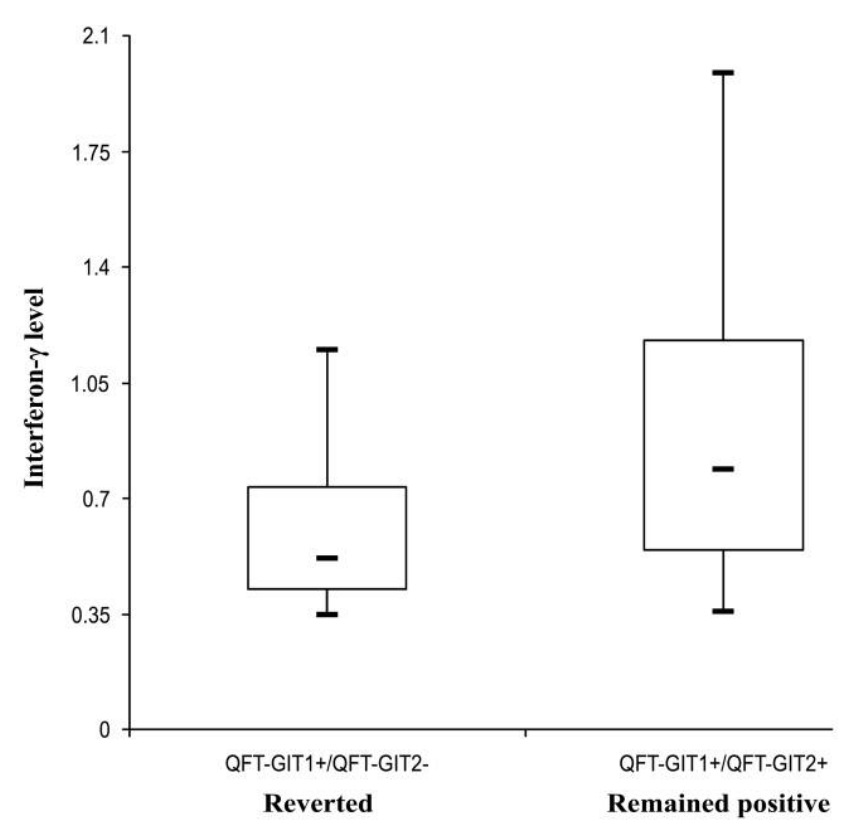

FIGURE 2. Interferon- $\gamma$ level comparison for healthcare workers whose QuantiFERON-TB Gold In-Tube (QFT-GIT; Cellestis) test results reverted from positive to negative (QFT-GIT1+/QFT-GIT2-) and for those whose results remained positive (QFT-GIT1+/QFTGIT2+).

results and negative QFT-GIT2 results) using an independent sample $t$ test. Similarly, the mean IFN- $\gamma$ levels for the HCWs with positive QFT-GIT1 and positive $h$ TST results was calculated and compared with HCWs who had positive QFTGIT1 and negative $h$ TST results using an independent sample $t$ test. Box plots were used to compare the IFN- $\gamma$ response level distribution among the aforementioned groups. We also compared the mean IFN- $\gamma$ levels between QFT-GIT1 and QFT-GIT2 in HCWs whose results reverted and whose results remained positive after repeated testing using a paired sample $t$ test. A $P$ value of less than .05 was considered to denote statistical significance. All calculations were done using SPSS software, version 17.0 (SPSS).

\section{RES U L T S}

A total of 6,530 HCWs were screened over a period of 1 year from January 1, 2008, to December 31, 2008, with the QFTGIT test. Of the 6,530 HCWs tested, a positive QFT-GIT test result was noted for $287(4.4 \%)$, a negative test result was noted for $6,243(95.5 \%)$, and an indeterminate result was noted for $58(0.88 \%)$ (Figure 1). Among the 287 HCWs who had positive QFT-GIT test results, only $123(42.7 \%)$ had a positive $h$ TST result. All $123 \mathrm{HCWs}$ underwent assessment, and active TB infection was ruled out. Of the remaining 164 HCWs, 146 had a negative $h$ TST results, 13 had unknown TST status, and 5 had a history of allergic reaction to TST. The conversion rate for LTBI with the QFT-GIT1 test was $2.5 \%$ (164 of $6,530 \mathrm{HCWs}$ ). This conversion rate of $2.5 \%$ was extremely high for our institution, compared with previous TST experience, and represented a 25 -fold increase. The typical conversion rate with TST at our institution has been less than $0.1 \%$ per year.

Of these 164 HCWs, 135 returned for follow-up and underwent complete assessment via the occupational health program, including history, physical examination, and chest radiography, to exclude active TB infection. The remaining 29 HCWs were lost to follow-up. Among the 135 HCWs, 130 had a negative $h$ TST result, 2 had unknown TST status, and $3 \mathrm{had}$ a history of allergic reaction to TST. The evaluation for active TB in all $135 \mathrm{HCWs}$ yielded negative results. In addition to this assessment, these 135 HCWs underwent QFTGIT2 testing, and 130 of the 135 had the TST performed at the same time. The TST was not performed for 3 HCWs because of allergy to TST and in 2 HCWs for unknown reasons.

The demographic characteristics of the 135 HCWs who underwent additional testing are listed in Table 1. The mean ages of women and men were 42.37 and 44.08 years, respectively. When the 135 HCWs underwent QFT-GIT2, 66 $(48.9 \%)$ had negative results at this time, and results remained positive for $69(51.1 \%)$ (Figure 1). This resulted in a reversion rate of $48.9 \%$ ( $95 \%$ confidence interval [CI], 40\%-57\%). Of the 130 HCWs who underwent the TST, only 2 had positive results, 124 had negative results, and 4 did not return for the test interpretation. The 2 HCWs who had positive TST results also had positive QFT-GIT2 results.

The mean IFN- $\gamma$ response level was calculated for those whose QFT-GIT results reverted to negative and for those whose results remained positive. The mean IFN $-\gamma$ response level for those whose results reverted was $0.69 \mathrm{IU}$ (95\% CI, $0.56-0.82$ ), whereas for those whose results remained positive,

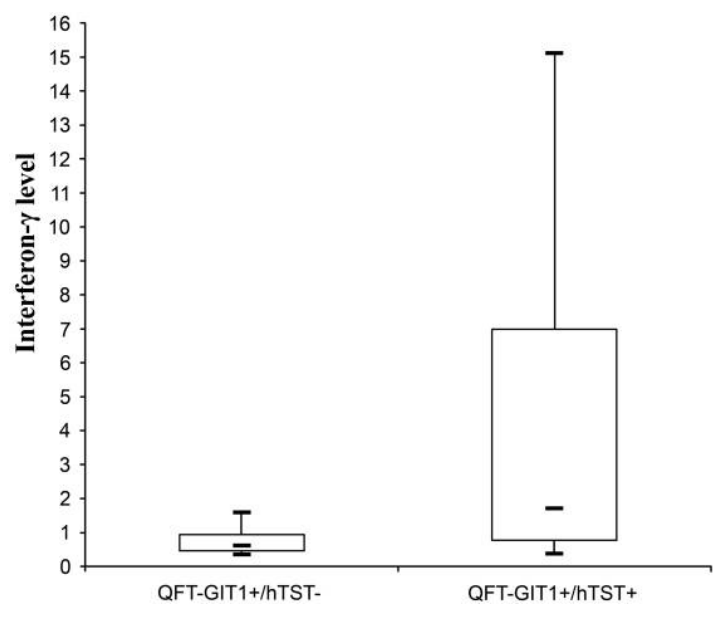

FIGURE 3. Interferon- $\gamma$ level comparison for 146 healthcare workers (HCWs) who had positive QuantiFERON-TB Gold In-Tube (QFT-GIT; Cellestis) test results and negative tuberculin skin test history ( $h \mathrm{TST}$ ) results (QFT-GIT1+/hTST-) versus $123 \mathrm{HCW}$ with positive results of both tests (QFT-GIT1+/hTST + ). 
TABLE 2. Interferon (IFN) $-\gamma$ Levels in Healthcare Workers Whose Results Reverted to Negative on Additional Testing

\begin{tabular}{|c|c|c|}
\hline \multirow[b]{2}{*}{ Serial number } & \multicolumn{2}{|c|}{ IFN- $\gamma$ level, IU/mL } \\
\hline & QFT-GIT1 & QFT-GIT2 \\
\hline 1 & 0.41 & -0.58 \\
\hline 2 & 0.46 & 0.07 \\
\hline 3 & 0.44 & 0.16 \\
\hline 4 & 0.48 & 0.17 \\
\hline 5 & 0.39 & 0.01 \\
\hline 6 & 3.05 & -0.39 \\
\hline 7 & 0.73 & 0.14 \\
\hline 8 & 0.70 & 0.00 \\
\hline 9 & 1.45 & 0.07 \\
\hline 10 & 0.46 & -0.01 \\
\hline 11 & 0.41 & 0.18 \\
\hline 12 & 0.42 & 0.20 \\
\hline 13 & 0.64 & -0.02 \\
\hline 14 & 0.48 & 0.10 \\
\hline 15 & 0.40 & 0.11 \\
\hline 16 & 1.82 & 0.23 \\
\hline 17 & 0.53 & 0.17 \\
\hline 18 & 0.42 & 0.14 \\
\hline 19 & 0.60 & 0.22 \\
\hline 20 & 0.37 & 0.16 \\
\hline 21 & 1.49 & 0.14 \\
\hline 22 & 1.15 & 0.00 \\
\hline 23 & 1.55 & 0.00 \\
\hline 24 & 0.43 & -0.01 \\
\hline 25 & 0.54 & 0.27 \\
\hline 26 & 0.92 & -0.01 \\
\hline 27 & 0.78 & 0.01 \\
\hline 28 & 0.58 & 0.21 \\
\hline 29 & 2.95 & 0.16 \\
\hline 30 & 0.53 & 0.06 \\
\hline 31 & 0.45 & 0.25 \\
\hline 32 & 0.71 & 0.05 \\
\hline 33 & 0.40 & 0.10 \\
\hline 34 & 0.36 & 0.00 \\
\hline 35 & 0.77 & 0.00 \\
\hline 36 & 0.66 & 0.26 \\
\hline 37 & 0.38 & 0.07 \\
\hline 38 & 0.37 & 0.14 \\
\hline 39 & 0.52 & 0.00 \\
\hline 40 & 0.52 & 0.15 \\
\hline 41 & 0.53 & 0.00 \\
\hline 42 & 0.51 & 0.05 \\
\hline 43 & 0.43 & 0.20 \\
\hline 44 & 0.41 & 0.14 \\
\hline 45 & 0.78 & 0.05 \\
\hline 46 & 0.43 & 0.00 \\
\hline 47 & 0.75 & 0.14 \\
\hline 48 & 0.35 & 0.26 \\
\hline 49 & 0.51 & 0.00 \\
\hline 50 & 0.37 & 0.10 \\
\hline
\end{tabular}

TABLE 2. (Continued)

\begin{tabular}{lcc}
\hline & \multicolumn{2}{c}{ IFN- $\gamma$ level, IU/mL } \\
\cline { 2 - 3 } Serial number & QFT-GIT1 & QFT-GIT2 \\
\hline 51 & 0.48 & 0.14 \\
52 & 0.75 & 0.14 \\
53 & 0.39 & 0.06 \\
54 & 0.87 & 0.24 \\
55 & 0.54 & 0.00 \\
56 & 0.66 & 0.10 \\
57 & 0.44 & 0.29 \\
58 & 0.35 & 0.00 \\
59 & 0.47 & 0.00 \\
60 & 0.58 & 0.00 \\
61 & 1.73 & 0.01 \\
62 & 0.46 & 0.28 \\
63 & 0.57 & 0.00 \\
64 & 0.44 & 0.16 \\
65 & 0.52 & 0.00 \\
66 & 0.86 & 0.02 \\
\hline NOTE. QFT-GIT1, initial QuantiFERON-TB Gold In- \\
Tube (Cellestis) test; QFT-GIT2, repeated QuantiFERON- \\
TB Gold In-Tube test. & & \\
& &
\end{tabular}

it was $1.28 \mathrm{IU}$ (95\% CI, 0.88-1.67). The difference in the mean values was statistically significant $(P=.007)$.

We also compared the mean IFN- $\gamma$ response level in HCWs who had positive results of both QFT-GIT1 and $h$ TST and in HCWs who had positive QFT-GIT1 results and negative $h$ TST results. The mean IFN- $\gamma$ response level in HCWs with positive $h$ TST results was 3.92 IU (95\% CI, 3.21-4.63), whereas HCWs with negative $h \mathrm{TST}$ results had a mean response level of $0.96 \mathrm{IU}$ (95\% CI, 0.76-1.16). Again, the difference in the mean values was statistically significant $(P<.001)$.

Then we compared the IFN- $\gamma$ response level distribution in HCWs whose QFT-GIT test results reverted from positive to negative with those whose results remained positive (Figure 2). A box plot was used for interpretation. We also compared the IFN- $\gamma$ level distribution in HCWs who had positive results of both QFT-GIT1 and $h$ TST with HCWs who had positive QFT-GIT1 test results and negative $h$ TST results (Figure 3).

On comparing the IFN- $\gamma$ levels (Tables 2 and 3 ) between QFT-GIT1 and QFT-GIT2, there was a significant difference $(P<.001)$ in the mean levels among the HCWs whose results reverted. The mean IFN- $\gamma$ level for QFT-GIT1 was $0.69 \mathrm{IU}$, and for QFT-GIT2 it was $0.08 \mathrm{IU}$. Among HCWs whose results did not revert, there was no significant difference $(P=.57)$ in mean IFN- $\gamma$ levels. The mean IFN- $\gamma$ level for QFT-GIT1 was 1.27 IU, and for QFT-GIT2 it was 1.19 IU.

Our institution's direct costs were $\$ 436,096$ for screening 6,530 HCWs with QFT-GIT, and the cost for the same HCWs if they had been screened with TST would have been $\$ 78,360$. 
TABLE 3. Interferon (IFN) $\gamma$ Levels in Healthcare Workers Whose Results Remained Positive on Additional Testing

\begin{tabular}{|c|c|c|}
\hline \multirow[b]{2}{*}{ Serial number } & \multicolumn{2}{|c|}{ IFN- $\gamma$ level, IU/mL } \\
\hline & QFT-GIT1 & QFT-GIT2 \\
\hline 1 & 1.92 & 6.36 \\
\hline 2 & 0.92 & 0.85 \\
\hline 3 & 0.70 & 0.80 \\
\hline 4 & 0.79 & 0.49 \\
\hline 5 & 1.10 & 0.43 \\
\hline 6 & 0.38 & 0.41 \\
\hline 7 & 0.56 & 0.44 \\
\hline 8 & 0.92 & 0.42 \\
\hline 9 & 1.14 & 0.87 \\
\hline 10 & 6.93 & 2.82 \\
\hline 11 & 5.19 & 6.41 \\
\hline 12 & 1.99 & 3.18 \\
\hline 13 & 0.38 & 0.64 \\
\hline 14 & 0.45 & 0.36 \\
\hline 15 & 0.84 & 0.44 \\
\hline 16 & 0.73 & 0.41 \\
\hline 17 & 0.45 & 0.36 \\
\hline 18 & 2.17 & 1.24 \\
\hline 19 & 1.08 & 1.53 \\
\hline 20 & 1.09 & 1.82 \\
\hline 21 & 1.11 & 1.64 \\
\hline 22 & 1.50 & 0.98 \\
\hline 23 & 0.46 & 1.06 \\
\hline 24 & 1.09 & 0.49 \\
\hline 25 & 0.77 & 0.52 \\
\hline 26 & 0.68 & 0.48 \\
\hline 27 & 0.38 & 0.65 \\
\hline 28 & 0.94 & 1.61 \\
\hline 29 & 0.58 & 0.42 \\
\hline 30 & 1.56 & 0.52 \\
\hline 31 & 9.29 & 4.51 \\
\hline 32 & 0.59 & 0.47 \\
\hline 33 & 1.20 & 1.16 \\
\hline 34 & 1.23 & 0.67 \\
\hline 35 & 0.46 & 0.62 \\
\hline 36 & 0.38 & 0.49 \\
\hline 37 & 0.82 & 0.47 \\
\hline 38 & 0.75 & 0.54 \\
\hline 39 & 0.73 & 0.82 \\
\hline 40 & 0.79 & 0.47 \\
\hline 41 & 0.97 & 1.20 \\
\hline 42 & 1.59 & 1.11 \\
\hline 43 & 1.38 & 0.51 \\
\hline 44 & 0.43 & 0.37 \\
\hline 45 & 0.44 & 0.41 \\
\hline 46 & 1.43 & 1.54 \\
\hline 47 & 2.10 & 1.86 \\
\hline 48 & 0.95 & 1.22 \\
\hline 49 & 0.53 & 0.48 \\
\hline 50 & 1.77 & 1.43 \\
\hline
\end{tabular}

TABle 3. (Continued)

\begin{tabular}{lcc}
\hline & \multicolumn{2}{c}{ IFN- $\gamma$ level, IU/mL } \\
\cline { 2 - 3 } Serial number & QFT-GIT1 & QFT-GIT2 \\
\hline 51 & 0.79 & 0.59 \\
52 & 1.72 & 3.71 \\
53 & 0.58 & 0.71 \\
54 & 0.62 & 1.44 \\
55 & 0.47 & 0.83 \\
56 & 0.38 & 0.76 \\
57 & 0.56 & 0.49 \\
58 & 0.76 & 2.18 \\
59 & 0.45 & 0.92 \\
60 & 0.47 & 0.43 \\
61 & 0.63 & 0.43 \\
62 & 1.16 & 1.70 \\
63 & 0.97 & 0.97 \\
64 & 0.83 & 0.87 \\
65 & 8.41 & 3.11 \\
66 & 1.06 & 1.73 \\
67 & 0.36 & 0.49 \\
68 & 0.49 & 0.49 \\
69 & 0.69 & 1.19 \\
\hline
\end{tabular}

Note. QFT-GIT1, initial QuantiFERON-TB Gold InTube (Cellestis) test; QFT-GIT2, repeated QuantiFERONTB Gold In-Tube test.

In addition to the screening costs, other indirect costs were observed because of the need for more intense testing and follow-up visits for HCWs with positive QFT-GIT test results. This included performing confirmatory TSTs, additional chest radiographs, extra nurse assessments, and supplemental provider examinations. Our total direct cost outlay was $\$ 521,890$ for 6,530 HCWs, resulting in a per-person cost of $\$ 79.92$ per HCW.

\section{I S C USS I ON}

To our knowledge, this is the first study performed in the United States to completely replace the TST with QFT-GIT for screening of HCWs in such a massive scale. Although the QFT-GIT test has many attractive features, our study results demonstrate the problems associated with it when used as the sole screening test. We had an unexpectedly high number of LTBI-positive results with the QFT-GIT, compared with the TST. We had expected that the QFT-GIT would reduce overestimations of LTBI; instead, our study yielded the opposite result, with a 25 -fold increase in the number of LTBI cases, compared with TST. This concern was mentioned in the Canadian Tuberculosis Standards, 2007 edition, which noted, "Use of IGRA in screening low-prevalence populations may lead to a significant number of positive IGRA tests. The significance of such positive tests in a population at low risk 
is not yet known; this will make interpretation and management difficult." ", p71 The reason for a high positive rate with QFT-GIT in our HCWs is unclear. One possibility to consider is whether recurrent TST testing as a part of routine annual screening led to development of a positive QFT-GIT test result. The other problem was high reversion rate $(48.9 \%)$ with QFT-GIT in this study, supporting concerns about the reproducibility of results with serial testing. ${ }^{10}$ Our results show that HCWs whose results reverted had a mean IFN- $\gamma$ level of $0.69 \mathrm{IU} / \mathrm{mL}$, which is close to the cutoff value $(0.35 \mathrm{IU} /$ $\mathrm{mL}$ ), compared with a mean IFN- $\gamma$ level of $1.28 \mathrm{IU} / \mathrm{mL}$ for those whose results remained positive. These results agree with previous studies which demonstrated that reversion rates are high when there is discordance between TST and QFTGIT and when the IFN- $\gamma$ responses are close to the cutoff value. ${ }^{11}$ Our results also demonstrate disagreement between the two screening tests (TST and QFT-GIT). So, in the absence of the gold standard test for LTBI, the disagreement between QFT-GIT and TST along with high reversion rate with QFT-GIT raise significant concerns about the effectiveness of QFT-GIT as sole screening test. The high conversion rate not only lead to fear and confusion, but it also resulted in significant costs, time, and evaluation-related work for positive cases. The other problem was the high reversion rate among these converters when the QFT-GIT was repeated, with a significant difference in the mean IFN- $\gamma$ levels between QFT-GIT1 and QFT-GIT2 (0.69 vs $0.08 \mathrm{IU} / \mathrm{mL})$. So, what to make of these reversions?

Another important result in our study is that there is statistically significant difference in mean IFN- $\gamma$ level between HCWs who had positive results of both QFT-GIT1 and $h$ TST tests and those who had positive QFT-GIT1 test results and negative $h$ TST results (3.92 vs 0.96 ). When the distribution of IFN- $\gamma$ level was compared (Figure 3), we noticed that, in more than $50 \%$ of HCWs who had positive results of both tests, the level was greater than $1 \mathrm{UL} / \mathrm{ml}$, whereas in HCWs with positive QFT-GIT1 test results and negative $h$ TST results, more than $50 \%$ of had a level less than $1 \mathrm{UL} / \mathrm{mL}$. Similarly, when the distribution of IFN- $\gamma$ level was compared for HCWs whose results reverted versus those whose results remained positive (Figure 2), the HCWs whose results reverted had results close to the cutoff value. This raises the question of whether we need a higher cutoff value for a positive QFTGIT test result, given the low prevalence of TB in the United States. Higher cutoff values in low-prevalence populations may decrease the high positive QFT-GIT test result rate and, at the same time, decrease the reversion rate.

The next issue is the cost effectiveness of QFT-GIT. Some studies demonstrate that using QFT-GIT in place of TST is cost effective ${ }^{12}$ but others demonstrate that the most costeffective use of QFT-GIT is to test TST-positive persons. ${ }^{13} \mathrm{As}$ mentioned in our results section; we had increased cost which was not anticipated as we were expecting a lower number of positives with QFT-GIT. With our experience, we conclude that the most cost effective strategy is to test TST positive persons with QFT-GIT rather than completely replacing the TST with QFT-GIT in screening HCWs, especially in low TB prevalent regions of the world. With these outcomes, our institution decided to go back to the TST method for annual screening instead of using the QFT-GIT.

The main limitation of this study is its focus on the data for subjects with positive QFT-GIT1 test results and negative $h \mathrm{TST}$ results; it does not focus on subjects with positive $h \mathrm{TST}$ results and negative QFT-GIT1 results, which we did not investigate. The second limitation is that we compared QFTGIT performance with TST performance in the absence of a gold standard test for LTBI. There is no gold standard test for LTBI. The third limitation is that the QFT-GIT results were not retested using the same specimen in the laboratory. Another limitation is that we did not follow up on HCWs who had indeterminate results. Their status may have been clarified if they had undergone additional QFT-GIT testing.

This large-scale study shows that QFT-GIT holds promise in helping supplement TB screening in healthcare settings, but it does not show promise in fully replacing the traditional TST screening method. At this time, many healthcare organizations are wondering whether they should replace their traditional TST with QFT-GIT methodology in TB screening of HCWs. Our outcomes with QFT-GIT versus TST do not support such a change because of the high cost and variability of serial QFT-GIT testing. This is especially true in the first year of switching from TST to QFT-GIT. There is a significant cost and clinical learning curve that needs to be overcome when understanding cutoff levels in low prevalence populations. QFT-GIT may be more appropriately used as an additional tool when trying to make clinical decisions in highrisk populations, such as BCG-vaccinated individuals from locations where TB is endemic. More prospective studies need to be performed to know the true effectiveness of QFT-GIT as a screening tool, especially in populations with a low prevalence of TB.

\section{ACKNOWLEDGMENTS}

Potential conflicts of interest. All authors report no conflicts of interest relevant to this article.

Address reprint requests to Sumanth Gandra, MD, MPH, 41 Sheridan Drive \#1, Shrewsbury, MA 01545 (gandrasatyam@gmail.com).

\section{REFERENCES}

1. Menzies D. What does tuberculin reactivity after Bacille Calmette-Guerin vaccination tell us? Clin Infect Dis 2000;31(suppl 3):S71-S74.

2. Andersen P, Munk ME, Pollock JM, Doherty TM. Specific immunebased diagnosis of tuberculosis. Lancet 2000;356(9235):1099-1104.

3. Centers for Disease Control and Prevention. Tuberculosis (TB). September 29, 2010. http://www.cdc.gov/tb/publications/factsheets/testing/QFT .htm/. Accessed January 29, 2010.

4. Menzies D, Pai M, Comstock G. Meta-analysis: new tests for the diagnosis 
of latent tuberculosis infection: areas of uncertainty and recommendations for research. Ann Intern Med 2007;146(5):340-354.

5. Mazurek GH, Jereb J, LoBue P, Iademarco MF, Metchock B, Vernon A; Division of Tuberculosis Elimination, National Center for HIV, STD, and TB Prevention, Centers for Disease Control and Prevention (CDC). Guidelines for using the QuantiFERON-TB Gold test for detecting $M y$ cobacterium tuberculosis infection, United States. MMWR Recomm Rep 2005;54(RR-15):49-55.

6. Nienhaus A, Schablon A, Le Bâcle C, Siano B, Diel R . Evaluation of the interferon- $\gamma$ release assay in healthcare workers. Int Arch Occup Environ Health 2008;81:295-300.

7. Harada N, Nakajima Y, Higuchi K, Sekiya Y, Rothel J, Mori T. Screening for tuberculosis infection using whole blood interferon $\gamma$ and Mantoux testing among Japanese healthcare workers. Infect Control Hosp Epidemiol 2006;27:442-448.

8. Pollock NR, Campos-Neto A, Kashino S, et al. Discordant Quantiferon-
TB Gold test results among US healthcare workers with increased risk of latent tuberculosis infection: a problem or solution? Infect Control Hosp Epidemiol 2008;29(9):878-886.

9. Diagnosis of Tuberculosis Infection and Disease. Canadian tuberculosis standards. 6th ed. 2007:83-84.

10. Perry S, Sanchez L, Yang S, Agarwal Z, Hurst P, Parsonnet P. Reproducibility of QuantiFERON-TB Gold In-Tube assay. Clin Vaccine Immunol 2008;15(3):425-432.

11. Pai M, O’Brien R. Serial testing for tuberculosis: can we make sense of T cell assay conversions and reversions? PLoS Med 2007;4(6):e208.

12. de Perio MA, Tsevat J, Roselle GA, Kralovic SM, Eckman MH. Costeffectiveness of interferon gamma release assays vs. tuberculin skin tests in health care workers. Arch Intern Med 2009;169(2):179-187.

13. Oxlade O, Schwartzman K, Menzies D. Interferon-gamma release assays and TB screening in high-income countries: a cost-effectiveness analysis. Int J Tuberc Lung Dis 2007;11:16-26. 